\title{
Optimum Design of Kinematically Redundant Planar Parallel Manipulator Following a Trajectory
}

\author{
K. V. Varalakshmi and J. Srinivas
}

\begin{abstract}
This paper presents an optimum design approach for selection of base-joint locations of a kinematically redundant parallel linkage following a desired trajectory by minimizing the active joint torques. Kinematically redundant parallel mechanisms offer several advantages such as reduced singularity loci, high dexterity indices etc. However, in path tracking problems, selection of redundant joint positions is tedious procedure due to existence of multiple solutions for inverse kinematics. Some criteria can be employed to resolve the complexity of identifying these extra degrees of freedom. In present task, joint torque minimization is considered and the problem is resolved as a static analysis formulation. The static analysis gives joint torques for known applied wrench vector and resulting torque-norm which is a function of redundant degrees of freedom (DOF) is minimized using genetic algorithms (GA). Interactive computer programs are developed for kinematics and static analysis along with binary coded GA. Results are presented using a 6-DOF, 3-PRRR planar parallel mechanism following predefined trajectories.
\end{abstract}

Index Terms-Genetic algorithms, kinematic redundancy, parallel manipulator, torque minimization.

\section{INTRODUCTION}

Parallel manipulators have gained importance due to their higher rigidity and payload-to-weight ratio and lower inertia permitting larger accelerations than classical serial manipulators. However, they have some drawbacks such as limited and complicated workspaces with singularities, reduced dexterity, highly non-isotropic input/output relations and complex mechanisms due to kinematically constrained structure. Presence of singularity loci within the workspace is one of the most important issues in parallel manipulators. In such instances, the manipulator looses or gains one or more degrees of freedom (DOF) and will become hardly controllable. In order to avoid the singularity loci and increase the performance of the manipulator, redundancy is often introduced in [1], [2]. There are two common types of redundancies introduced in mechanisms: actuation redundancy and kinematic redundancy. In a kinematically redundant manipulators, extra active joints are added to manipulator so as to increase the mobility of the manipulator. Relatively, the kinematic redundancy increases the workspace, and improves dexterity. Over the last one decade, several

Manuscript received September 25, 2014; revised December 20, 2014 The paper was presented at International Conference on Mechanics and Mechatronics Research 2014 at Kaula Lumpur.

K. V. Varalakshmi and J. Srinivas are with the National Institute of Technology, Rourkela, Odisha-769008 India (e-mail: kv.varalakshmi@gmail.com,srin07@yahoo.co.in). researchers worked on the kinematically redundant planar parallel mechanisms. A family of new kinematically redundant planar parallel manipulators were introduced by Ebrahimi et al. [3] by adding three kinematically redundant links to 3-RRR linkage. In order to avoid the singularities in the workspace during path planning, kinematic redundancy can be used to by varying the geometrical parameters of a mechanism instead of changing the basic structure. Fundamental definitions related to the Jacobian analysis and optimization strategy for the redundant actuator positions for singularity avoidance were given by Kotlarski et al.[4]. An analytical approach for singularity-free region in the workspace was presented by Zarkandi [5] using 3-PRRR linkage. Kotlarski et al. [6] introduced an approach of actuation torque minimization to avoid singularities in workspace. Niemann et al. [7] presented a time-optimal optimization approach for the path planning of kinematically redundant mechanisms. Recently, various issues in synthesis and analysis of parallel manipulators such kinematics, dynamics and control were formulated as optimization problems. More recently, Kucuk [8] also illustrated the optimization issues for achieving lowest power consumption. Several optimization schemes such as fuzzy logic [9], neural networks (NN), and generic algorithms (GA) [10] have been applied. However, most of these works were related to fully-actuated non-redundant cases. Most recently, Boudreau and Nokleby [11] illustrated the joint torque minimization scheme for a 6-DOF, 3-PRPR planar mechanism with redundant joint position and velocity constraints. Here, sequential quadratic programming methodology was employed to solve the problem.

Optimizing the actuated joint torques of parallel manipulators by adjusting the geometrical parameters is a difficult and time-consuming task. However, non-conventional optimization techniques are the best alternative to obtain unique solutions in most of the cases. Present paper describes GA as an optimization tool for the selection of redundant joint locations of the kinematically redundant planar parallel manipulator. The objective function of joint torque is expressed in terms of these variables. The kinematics and statics of 3-PRRR redundant parallel manipulator is employed to describe the joint torques. The dexterity index and torque intensities at optimized locations are illustrated. Effectiveness of the methodology is validated for two different trajectories. The paper is organized as follows: Section II explains the description of the 3-PRRR redundant linkage; velocity analysis, joint-torque estimation steps from static analysis and dexterity. Section III gives the optimization routine for solving formulation. Section IV illustrates the results and discussion with two examples. 


\section{DESCRIPTION OF MECHANISM}

A 6-DOF kinematically redundant planar parallel manipulator mechanism is obtained by adding extra active base prismatic joints to the existing 3-RRR manipulator linkage. Fig. 1 shows the mechanism with three branches each having two links with actuated revolute joints $\mathrm{O}_{i}$ fixed to the sliding bases [12]. The mobile platform is connected to active joints through passive revolute joints $\mathrm{B}_{i}$. Base prismatic joints are considered to be sliding on the path of constant inclination $\alpha_{\mathrm{I}}$ bounded within the base triangular platform. If the vertices $\mathrm{A}_{i}=\mathrm{O}_{i}(i=1,2,3)$, then the configuration is conventional 3-RRR manipulator.

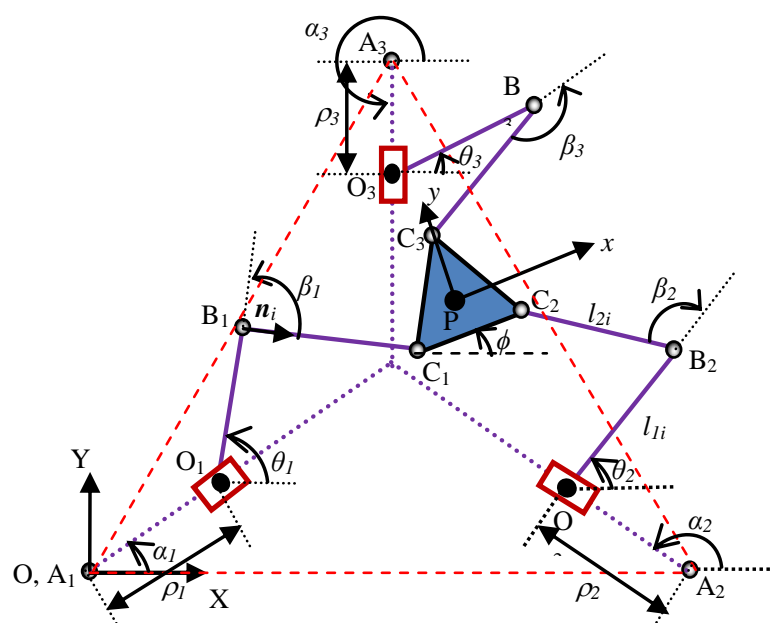

Fig. 1. 3-PRRR manipulator.

\section{A. Velocity Analysis}

The velocity analysis of $3-\underline{R R R}$ manipulator is first considered to simplify the velocity analysis of the 3-PRRR manipulator. The velocity equation shows the Jacobian matrices of the manipulators resulting from differentiating the non-linear loop-closure equations $f(\mathbf{q}, \mathbf{x})=0$ of the input $\mathbf{q}=\left[\theta_{1}, \theta_{2}, \theta_{3}\right]^{\mathrm{T}} \quad$ (joint displacements) and output $\mathbf{x}=[x, y, \phi]^{\mathrm{T}}$ (end-effector pose) variables, with respect to time, i.e.

$$
\mathbf{J}_{\mathbf{X}} \dot{\mathbf{x}}=\mathbf{J} \mathbf{q} \dot{\mathbf{q}}
$$

where $\mathbf{J}_{\mathbf{x}}=\partial \mathrm{f}(\mathbf{q}, \mathbf{x}) / \partial \mathbf{x}$ and $\mathbf{J}_{\mathbf{q}}=\partial \mathrm{f}(\mathbf{q}, \mathbf{x}) / \partial \mathbf{q}$ are $(m \times m)$ and $(m \times n)$ matrices respectively, $m$ is the DOFs in the Cartesian space and $n$ is the DOFs of the linkage (for non redundant manipulator $m=n$ ); While, $\dot{\mathbf{x}}$ and $\dot{\mathbf{q}}$ are the end-effector and joint velocities, respectively. The overall Jacobian matrix $\mathbf{J}$ can be expressed as:

$$
\mathbf{J}=\mathbf{J}_{\mathbf{x}}^{\mathbf{- 1}} \mathbf{J}_{\mathbf{q}}
$$

where $\mathbf{J}_{\mathbf{X}}$ and $\mathbf{J}_{\mathbf{q}}$ are direct and inverse Jacobian matrices respectively. Based on the inverse displacement, considering the geometry of the 3-RRR planar parallel manipulator in Fig. 1 , the loop closure equations can be written by defining the unit vector $\boldsymbol{n}_{i}$ directed along the distal links. Referring to Fig. 1 , the following relation can thus be obtained for each kinematic chain:

$$
l_{2 i} \boldsymbol{n}_{i}=\mathbf{O P}+[\mathbf{R}] P C_{i}^{\prime}-\mathbf{A B}_{i}
$$

By squaring on both sides of (3) and applying the law of cosines

$$
l_{2 i}^{2}=\left(\mathbf{O P}+[\mathbf{R}] P C_{i}^{\prime}-\mathbf{A B} \mathbf{B}_{i}\right)^{\mathrm{T}}\left(\mathbf{O P}+[\mathbf{R}] P C_{i}^{\prime}-\mathbf{A B} \mathbf{B}_{i}\right)
$$

where $[\mathbf{R}]=\left[\begin{array}{cc}\cos \phi & -\sin \phi \\ \sin \phi & \cos \phi\end{array}\right]$ is the rotation matrix, OP is the vector expressing point $\mathrm{P}$ and $\boldsymbol{P C _ { i } ^ { \prime }}$ is the vector from $\mathrm{P}$ to $\mathrm{C}_{i}$ expresses in the moving frame $\mathrm{xy}$.

Differentiating (4) with respect to time, the following relations can be obtained:

$$
l_{2 i} \boldsymbol{n}_{i}^{\mathrm{T}}\left[\begin{array}{c}
\dot{x} \\
\dot{y}
\end{array}\right]+\mathbf{Z}^{\mathrm{T}}\left(\boldsymbol{P} \boldsymbol{C}_{i} \times l_{2 i} \boldsymbol{n}_{i}\right) \dot{\phi}+l_{2 i} \boldsymbol{n}_{i}^{\mathrm{T}}\left[\begin{array}{c}
l_{1 i} \sin \theta_{i} \\
-l_{1 i} \cos \theta_{i}
\end{array}\right] \dot{\theta_{i}}=0
$$

Here $\boldsymbol{Z}$ is the unit vector along $\mathrm{z}$ direction. It can be expressed in matrix form for all three legs as:

$$
\left[\begin{array}{cc}
l_{21} \boldsymbol{n}_{1}^{\mathrm{T}} & \boldsymbol{Z}^{\mathrm{T}}\left(\boldsymbol{P} \boldsymbol{C}_{1} \times l_{21} \boldsymbol{n}_{1}\right) \\
l_{22} \boldsymbol{n}_{2}^{\mathrm{T}} & \boldsymbol{Z}^{\mathrm{T}}\left(\boldsymbol{P} \boldsymbol{C}_{2} \times l_{22} \boldsymbol{n}_{2}\right) \\
l_{23} \boldsymbol{n}_{3}^{\mathrm{T}} & \boldsymbol{Z}^{\mathrm{T}}\left(\boldsymbol{P} \boldsymbol{C}_{3} \times l_{23} \boldsymbol{n}_{3}\right)
\end{array}\right]\left[\begin{array}{c}
\dot{x} \\
\dot{y} \\
\dot{\boldsymbol{\phi}}
\end{array}\right]+\left[\begin{array}{ccc}
\lambda_{1} & 0 & 0 \\
0 & \lambda_{2} & 0 \\
0 & 0 & \lambda_{3}
\end{array}\right]\left[\begin{array}{c}
\dot{\theta}_{1} \\
\dot{\theta}_{2} \\
\dot{\theta}_{3}
\end{array}\right]=0
$$

where $\lambda_{i}=l_{1 i} l_{2 i} \boldsymbol{n}_{i}^{\mathrm{T}}\left(\sin \theta_{i}-\cos \theta_{i}\right)$

The (6) is similar to (1) representing the Jacobian matrices of 3-RRR planar parallel manipulator. The loop closure equations of 3-PRRR can be expressed from Fig.1 as:

$$
\begin{gathered}
l_{2 \mathrm{i}} \boldsymbol{n}_{i}=\mathbf{O P}+[\mathbf{R}] P \boldsymbol{C}_{i}^{\prime}-\mathbf{O B} \mathbf{B}_{i} \\
l_{2 i}^{2}=\left(\mathbf{O P}+[\mathbf{R}] \boldsymbol{P C}_{i}^{\prime}-\mathbf{O B}\right)^{\mathrm{T}}\left(\mathbf{O P}+[\mathbf{R}] \mathbf{P} \boldsymbol{C}_{i}^{\prime}-\mathbf{O B}_{i}\right)
\end{gathered}
$$

Differentiating (8) with respect to time results in:

$$
\begin{aligned}
l_{2 i} \boldsymbol{n}_{i}^{\mathrm{T}}\left[\begin{array}{c}
\dot{x} \\
\dot{y}
\end{array}\right] & +\boldsymbol{Z}^{\mathrm{T}}\left(\boldsymbol{P} \boldsymbol{C}_{i} \times l_{2 i} \boldsymbol{n}_{i}\right) \dot{\boldsymbol{\phi}} \\
& +l_{2 i} \boldsymbol{n}_{i}^{\mathrm{T}}\left[\begin{array}{c}
l_{l i} \sin \theta_{i} \\
-l_{l i} \cos \theta_{i}
\end{array}\right] \dot{\theta}_{i}-l_{2 i} \boldsymbol{n}_{i}^{\mathrm{T}} \dot{\rho}_{i}\left[\begin{array}{c}
\cos \alpha_{i} \\
\sin \alpha_{i}
\end{array}\right]=0
\end{aligned}
$$

It can be extended for all three legs and is expressed in matrix form as:

$$
\left[\begin{array}{ll}
l_{21} \boldsymbol{n}_{1}^{\mathrm{T}} & \boldsymbol{Z}^{\mathrm{T}}\left(\boldsymbol{P} \boldsymbol{C}_{1} \times l_{21} \boldsymbol{n}_{1}\right) \\
l_{22} \boldsymbol{n}_{2}^{\mathrm{T}} & \boldsymbol{Z}^{\mathrm{T}}\left(\boldsymbol{P C} \boldsymbol{C}_{2} \times l_{22} \boldsymbol{n}_{2}\right) \\
l_{23} \boldsymbol{n}_{3}^{\mathrm{T}} & \boldsymbol{Z}^{\mathrm{T}}\left(\boldsymbol{P} \boldsymbol{C}_{3} \times l_{23} \boldsymbol{n}_{3}\right)
\end{array}\right]\left[\begin{array}{c}
\dot{x} \\
\dot{y} \\
\dot{\phi}
\end{array}\right]+\left[\begin{array}{cccccc}
\xi_{1} & \lambda_{1} & 0 & 0 & 0 & 0 \\
0 & 0 & \xi_{2} & \lambda_{2} & 0 & 0 \\
0 & 0 & 0 & 0 & \xi_{3} & \lambda_{3}
\end{array}\right]\left[\begin{array}{c}
\dot{\rho}_{1} \\
\dot{\theta}_{1} \\
\dot{\rho}_{2} \\
\dot{\theta}_{2} \\
\dot{\rho}_{3} \\
\dot{\theta}_{3}
\end{array}\right]=0
$$

where, $\xi_{i}=-l_{2 i} \boldsymbol{n}_{i}^{\mathrm{T}}\left(\cos \alpha_{i}+\sin \alpha_{i}\right) \quad(i=1,2,3)$.

Comparing with (1), the Jacobian matrices of redundant 3-PRRR planar parallel manipulator can be derived. Further, the Jacobian matrices are dimensionally homogenized to avoid the conflicts with units. The developed equations allow the computation of the velocities of revolute and prismatic actuators for both the non-redundant and redundant manipulators when the velocity of the moving platform is 
known. The velocities of the base actuators are determined numerically during the optimization and used in providing velocity constraints. Under static conditions the relationship between the joint forces/torques and the end-effector wrench is [13]:

$$
\boldsymbol{F}=\mathbf{J}^{-\mathrm{T}} \boldsymbol{\tau}
$$

For a specified end-effector wrench $\boldsymbol{F}$ in (11) the generalized actuation joint torques/forces are given by:

$$
\boldsymbol{\tau}=\mathbf{J}^{\mathrm{T}} \boldsymbol{F}
$$

\section{B. Dexterity}

To measure the dexterity indices and to describe the overall kinematic behaviour of the manipulator condition number of Jacobian matrix has been used and also for evaluating the velocity, accuracy and rigidity mapping characteristics between the joint variables and the moving platform [14].

The condition number $\kappa$ is defined as

$$
1 \leq \kappa=\frac{\lambda_{2}}{\lambda_{1}} \leq \infty
$$

where $\lambda_{1}$ and $\lambda_{2}$ are the minimum and maximum singular values of the Jacobian matrix associated with a given posture. The condition number $\kappa$ ranges from 1 to $\infty$. In a pose where $\kappa$ is equal to 1 , the dexterity of the manipulator is considered to be best (isotropy) and it is dependent on manipulator configuration. Its reciprocal $1 / \kappa(0 \leq 1 / \kappa \leq 1)$ is referred as the conditioning index.

\section{FORMULATION OF OPTIMIZATION}

The optimization problem described as the determination of the optimum base prismatic link locations to minimize the actuated joint torques subjected to the kinematic and geometric constrains. Mathematically, it is expressed as:

Minimize

$$
\mathrm{T}\left(\rho_{i}\right)=\sqrt{\sum_{i=1}^{3} \tau_{i}^{2}}
$$

Subject to

$$
\begin{gathered}
\rho_{i, \text { min }} \leq \rho_{i} \leq \rho_{i, \text { max }} \\
\dot{\rho}_{i} \in\left[\dot{\rho}_{i, \text { min }}, \dot{\rho}_{i, \text { max }}\right] \\
\tau_{i} \leq \tau_{i, \text { max }}
\end{gathered}
$$

where $\rho_{i, \text { min }}, \dot{\rho}_{i, \text { min }}$ and $\rho_{i, \text { max }}, \dot{\rho}_{i, \text { max }}$ signify the lower and upper joint limits of positions and velocities that ensure the link- interference-free trajectories for the manipulator. $\tau_{i, \max }$ demonstrates the upper bounds of the torques that the actuators of the manipulator possess. The aim of kinematic design approach is to discover the optimal geometric configuration according to objective function. Specially, optimization-based kinematic synthesis is one of the significant steps in the design process to ensure high performance of the parallel manipulator with maximum stiffness and dexterity within the workspace. Here, the significance of optimization is to minimize the actuated joint torques. The optimization procedure employed in the present work is shown in Fig. 2.

To solve the minimization problem, an interactive binary coded GA optimization scheme is implemented in MATLAB. Derived from Darwin's theory of mutation, GA scheme employs three stages: selection, crossover and mutation. An initial population is randomly selected and the crossover and mutation operators are applied to the two strings at a time to produce two new strings for the next generation. More details of the methodology can be found in open literature [15]. In present work, an optimum population size of 30 is selected with crossover and mutation probabilities as 0.999 and 0.001 respectively. Population of points (locations of prismatic joints) are randomly generated in each time step of the trajectory and these individuals of a population are evaluated for minimization of objective function.

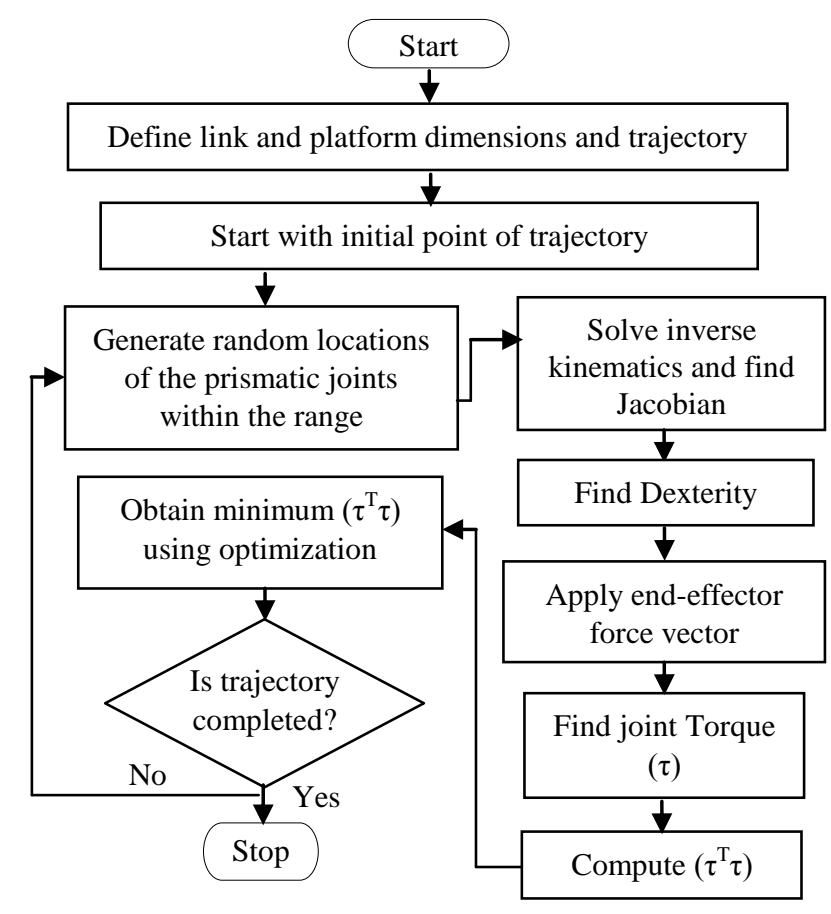

Fig. 2. Flowchart for optimization procedure.

\section{RESUlts AND DISCUSSION}

An optimization-based methodology has been developed for resolving the joint torques of kinematically-redundant planar parallel manipulator following a desired trajectory by choosing the maximum dexterity. To show the effectiveness of the optimization two trajectories are chosen. The actuator joint torques and the corresponding dexterity was computed numerically at each time step. The results were obtained for the non-redundant and redundant manipulators. The dimensional parameters chosen for the manipulator are $\mathrm{O}_{i} \mathrm{O}_{j}=0.5 \mathrm{~m}$ and $\mathrm{B}_{i} \mathrm{~B}_{j}=0.2 \mathrm{~m}, l_{1 i}=l_{2 i}=0.2 \mathrm{~m}$ and $\phi=0^{\circ}$. The base prismatic joint limits of the kinematically redundant 3-PRRR planar parallel manipulator were considered in the range of $0.05 \mathrm{~m}$ to $0.1 \mathrm{~m}$ for the optimization. The range is selected in such a way that the existence of workspace is feasible in all the limits. 


\section{Case 1: Straight-Line Path}

A straight- line trajectory along the $\mathrm{x}$-axis is considered in which the end-effector is moving towards right i.e., $(0.25 \mathrm{~m}$, $0.144 \mathrm{~m})$ to $(0.48 \mathrm{~m}, 0.144 \mathrm{~m})$ with an increment of $1.0 \mathrm{~mm}$. And also the desired trajectory passes singularity loci from $(0.405 \mathrm{~m}, 0.144 \mathrm{~m})$ to $(0.48 \mathrm{~m}, 0.144 \mathrm{~m})$ as can be seen from Fig. 3. The straight line trajectory and the singular points are shown within the constant orientation workspace of the non-redundant 3-RRR manipulator in Fig. 3. Jacobian matrices at each location corresponding to a set of initial base prismatic joint positions are obtained using user interactive modular based program. A constant force of $46.46 \mathrm{~N}$ is applied on the platform in the direction opposite to the motion so that the external wrench vector can be written as: $\mathrm{F}=\left[\begin{array}{lll}-46.46 & 0.0 & 0.0\end{array}\right]^{\mathrm{T}}$.

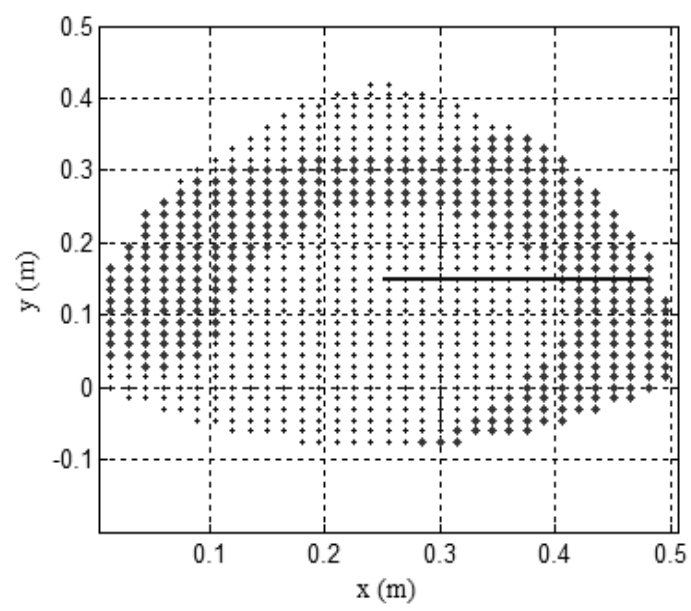

Fig. 3. Workspace and straight-line trajectory.

Fig. 4 shows the generalized torques required for the non-redundant 3-RRR manipulator. In theory, the joint torques should reach infinite values at singularity points. The finite values shown in the Fig. 4 are due to the displacement step chosen, which was $1.0 \mathrm{~mm}$. With a finer displacement step, the computation would have failed when the manipulator is exactly at the singularity loci. It is clear that the non-redundant manipulator cannot perform the desired trajectory tracking, as it requires infeasible generalized torques at the singularity loci.

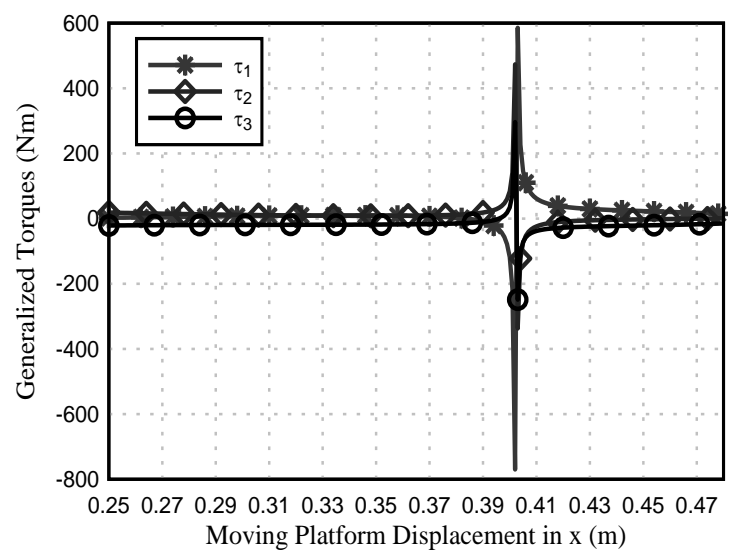

Fig. 4. Actuator generalized torques for the 3 -RRR manipulator for the Straight-line trajectory.

Fig. 5 shows the dexterity index of non-redundant and redundant manipulators; it is observed that in the non-redundant case, the dexterity is relatively low at singularity loci. More the dexterous, more will be freedom to cross the singularities. Fig. 6 shows a plot of the fitness versus number of generations at one of the trajectory points. It is seen that the convergence to fitness on average sense is achieved within 100 cycles. The computer time taken for converge is 20 seconds on a X86-based PC with 4 GB RAM and $3 \mathrm{GHz}$ dual core Intel processor. Fig. 7 shows the corresponding optimum set of prismatic joint locations at each point of a straight-line trajectory after the end of program execution. Fig. 8 shows the optimized torques for the redundant 3-PRRR manipulator. It clearly shows that the joint torques of the redundant manipulator are relatively lower, leading it cover the singularities without much difficulty, while maintaining actuator joint torques limitations. The intension is not to illustrate the reduction of joint torques due to redundancy, but the optimization outcomes from best prismatic joint positions.
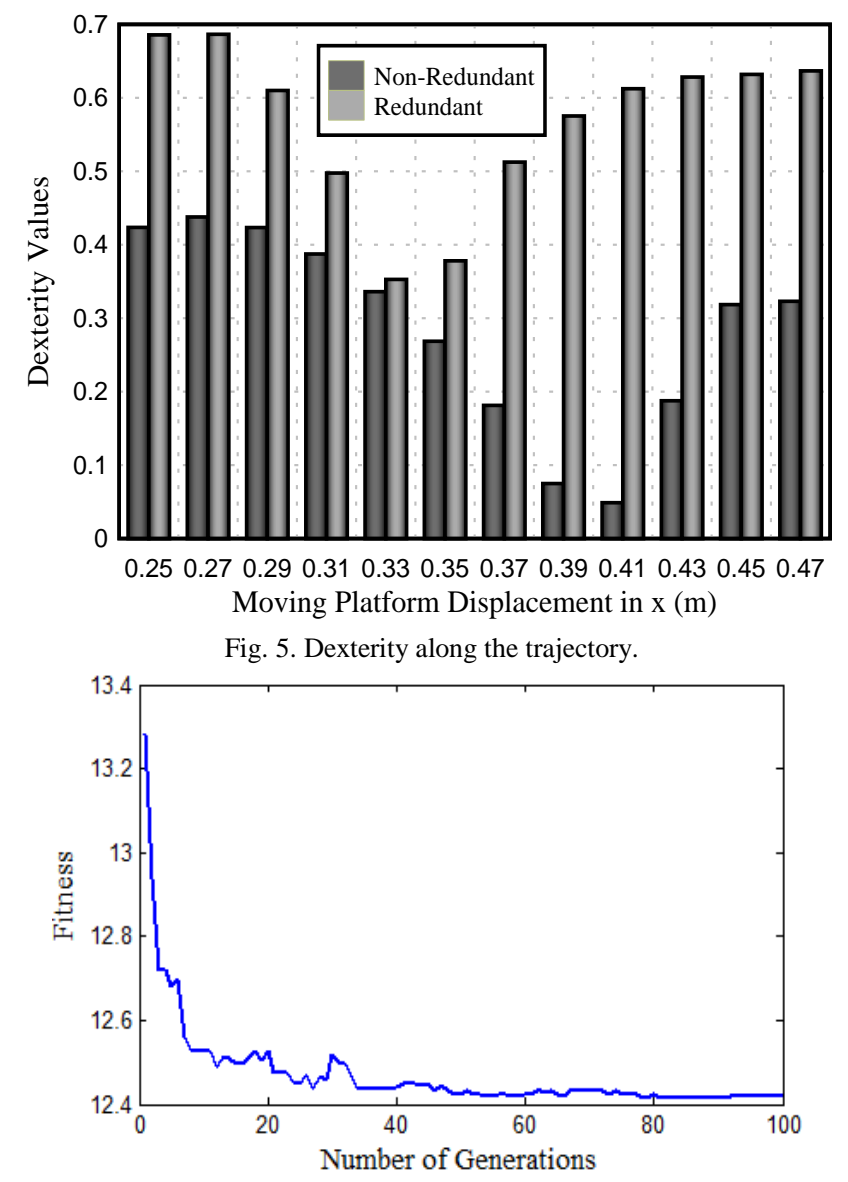

Fig. 6. The evolution of Torques at one of the trajectory points.

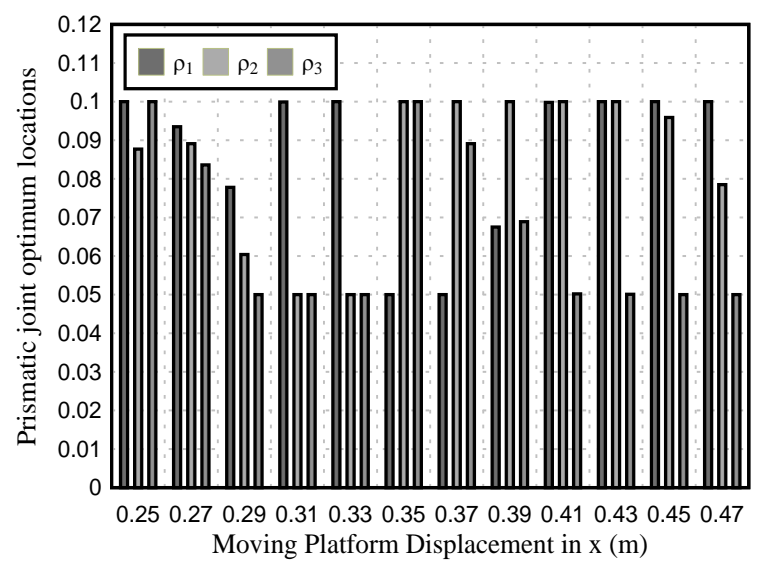

Fig. 7. Optimum locations of prismatic joints. 


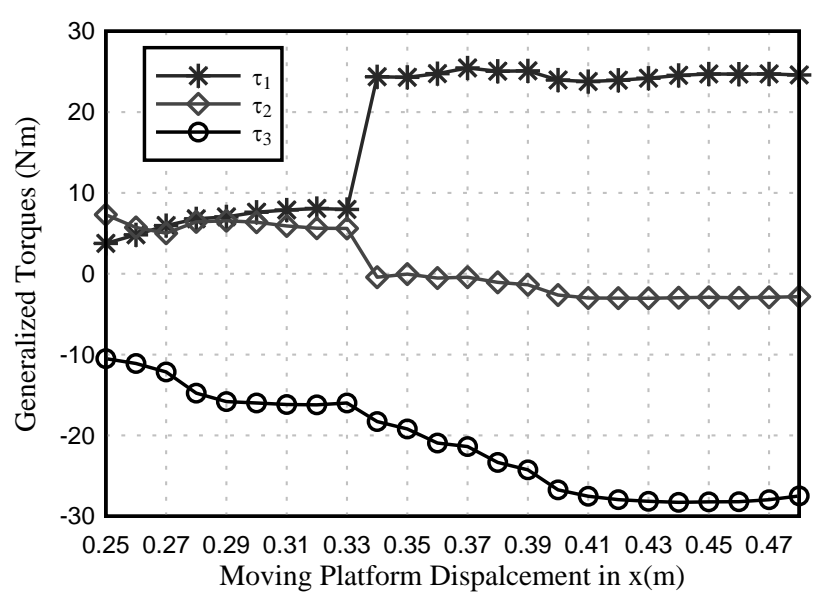

Fig. 8. Actuator optimized torques for the 3-PRRR manipulator.

Fig. 9 shows the base prismatic joint forces of 3-PRRR manipulator.

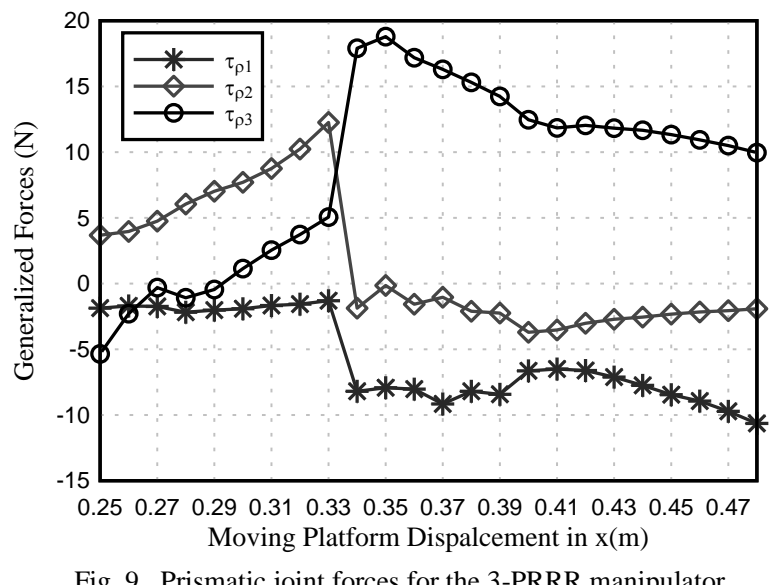

\section{Case 2: Butterfly Path}

The chosen second trajectory is a logarithmic spiral described by the following equations [8]:

$$
x=r \cos (a \pi t), y=r \sin (b \pi t)
$$

where $x, y$ are the coordinates and $r$ is radius (in present case: $r=0.15 \mathrm{~m})$; the constants $a=0.4 \mathrm{~m}, b=0.8 \mathrm{~m}$ and time $t \in[0,5$ seconds] with increment 0.025 second. The wrench applied at the platform is defined by: $\mathrm{F}=[-46.46 \times a \sin (a \pi t)$, $46.46 \times b \cos (b \pi t), 4.646]^{\mathrm{T}}$ N. Fig. 10 shows the path and singularity points (red in colour) within a constant orientation workspace.

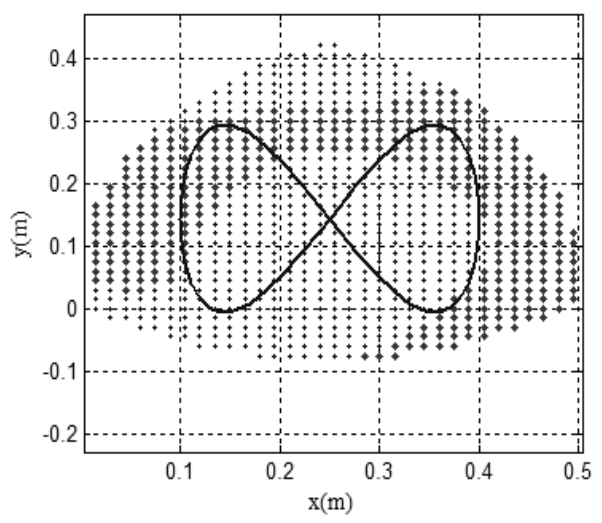

Fig. 10. Workspace and butterfly trajectory.

Fig. 11 shows the generalized torques along the proposed butterfly trajectory in each time step of a non-redundant 3-RRR manipulator (without optimization).

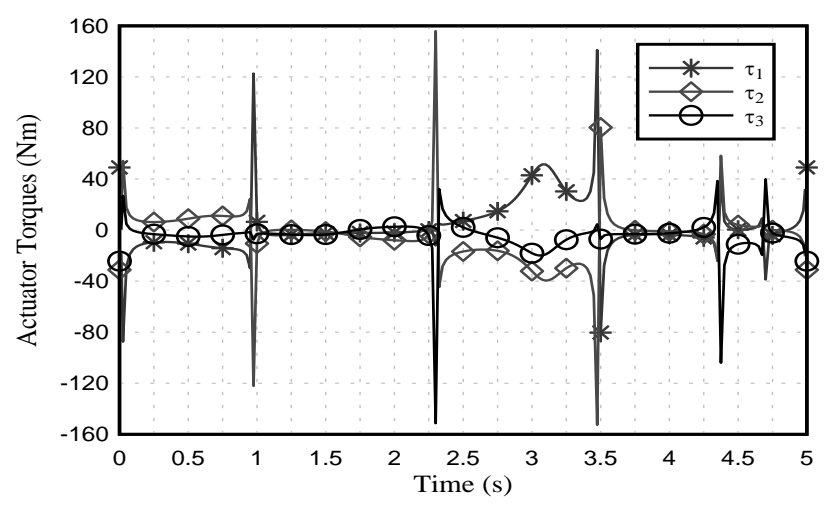

Fig. 11. Actuator generalized torques for the 3-RRR manipulator.

Fig. 12 shows the dexterity of non-redundant and redundant manipulators; here in the non-redundant case the dexterity is very much low at singularity points.

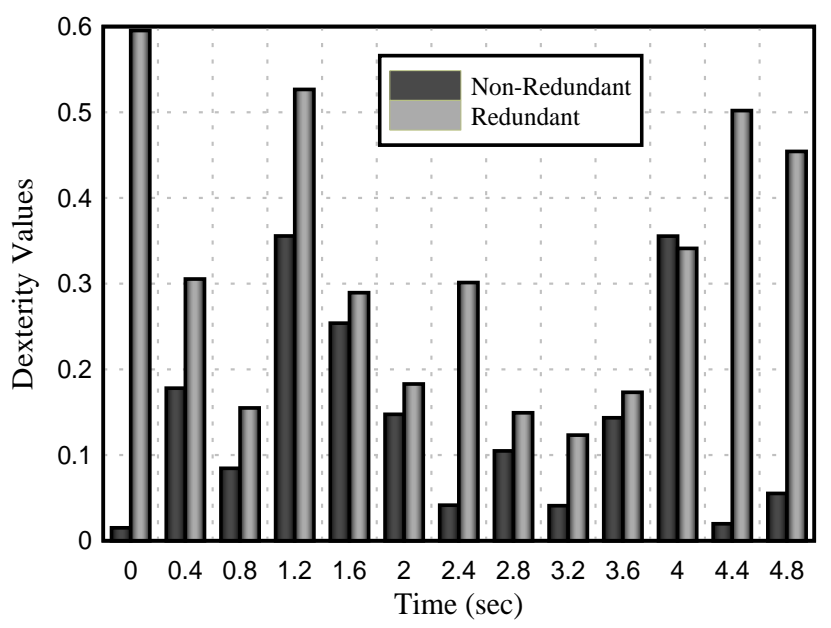

Fig. 12. Dexterity along the trajectory.

Fig. 13 shows the prismatic joint optimum locations where the joint torques reach minimum in each time step.

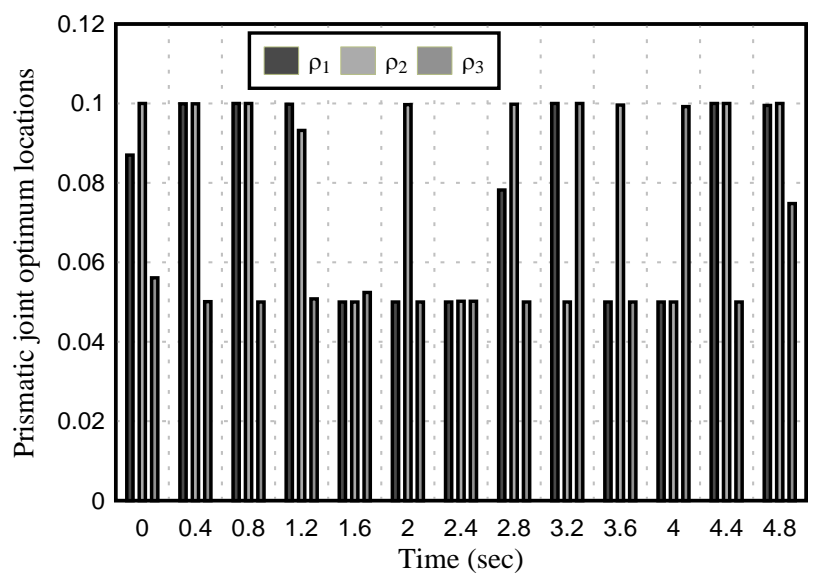

Fig. 13. Optimum locations of prismatic joints at each trajectory point.

Fig. 14 shows the generalized torques after optimization. It is observed that the torque amplitudes are relatively less. Comparing Fig. 11 with Fig. 14, it can be seen that the redundant manipulator easily passes through the singular configuration, while maintaining feasible actuated joint torques. 


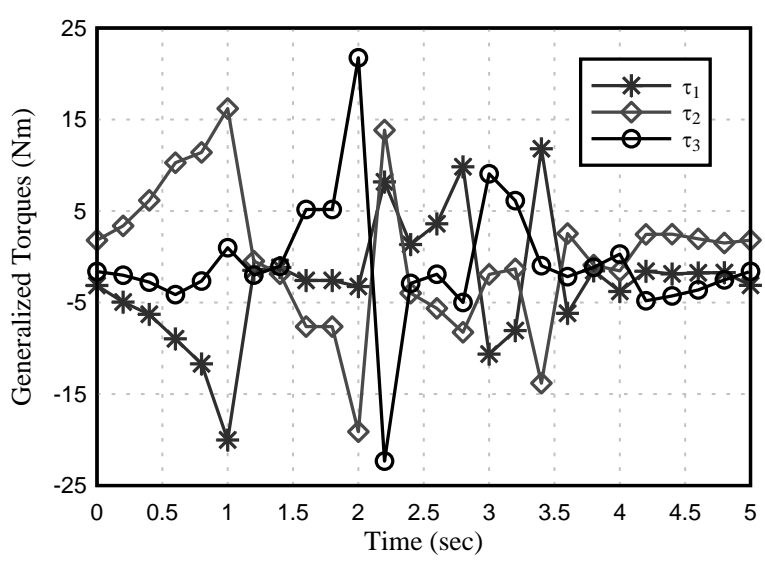

Fig. 14. Actuator optimized torques for the 3-PRRR manipulator.

The obtained base prismatic joint forces of 3-PRRR manipulator are shown in Fig. 15. It is seen that these values are relatively small compared to un-optimized redundant case as well as non-redundant case. Unconstrained optimization through GA has given excellent outcomes in short time.

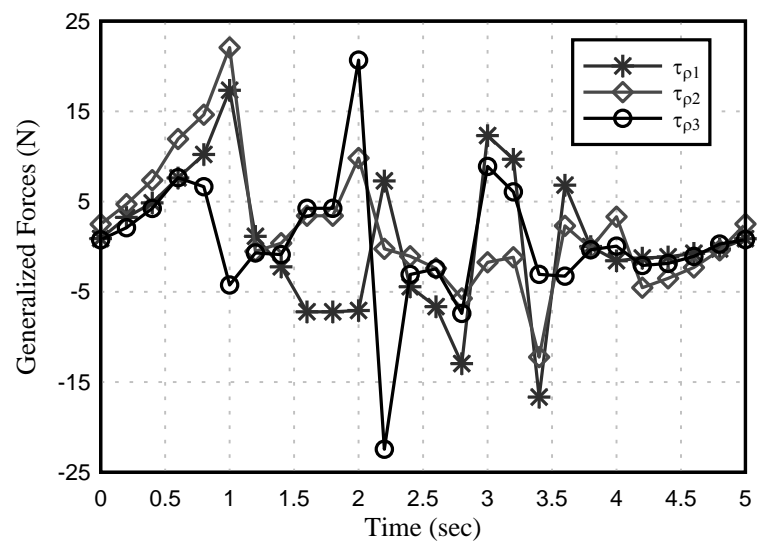

Fig. 15. Prismatic joint forces for the 3-PRRR manipulator.

\section{CONClusions}

This paper presented an optimization based methodology for the kinematically-redundant 3-PRRR planar parallel manipulator for achieving minimum actuated joint torques while following a desired trajectory. The active revolute joint torques were explicitly expressed in terms of redundant joint positions and the problem was casted as an optimization formulation with kinematic and geometric constraints. Results of the analysis clearly show that the kinematically-redundant manipulator has improved performance over the non-redundant for the selected trajectories. Also, the genetic algorithms based optimization scheme has given good results compared to randomly selected base joint locations of the redundant mechanism. This work forms a road for optimum design of redundant manipulators towards energy minimization. It can be extended with additional performance indices such as dexterity and stiffness so as to explore with further practicality.

\section{REFERENCES}

[1] S. Lee and S. Kim, "Kinematic analysis of generalized parallel manipulator systems," in Proc. IEEE Conference on Decision and Control, San Antonio: TX, USA, 1993, pp. 1097-1102.
[2] J. P. Merlet, "Redundant parallel manipulators," Journal of Laboratory Robotic and Automation, vol. 8, no. 1, pp. 17-24, 1996.

[3] I. Ebrahimi, I. J. A. Carretero, and R. Boudreau, "3-PRRR redundant planar parallel manipulator: inverse displacement, workspace and singularity analyses," Mechanism and Machine Theory, vol. 42, no. 8, pp. 1007-1016, 2007.

[4] J. Kotlarski, H. Abdellatif, and B. Heimann, "Improving the pose accuracy of a planar 3-RRR parallel manipulator using kinematic redundancy and optimized switching patterns," in Proc. IEEE Int. Conference on Robotics and Automation, Pasadena, CA, USA, 2008, pp. 3863-3868.

[5] S. Zarkandi, "Singularity analysis of a 3-PRRR kinematically redundant planar parallel manipulator," Journal of Mechanical Engineering, vol. 41, no. 1, pp.1-6, 2010.

[6] J. Kotlarski, T. Do Thanh, H. Abdellatif, and B. Heimann, "Singularity avoidance of a kinematically redundant parallel robot by a constrained optimization of the actuation forces," in Proc. 17th CISM-IFToMM Symposium on Robot Design, Dynamics, and Control, Tokyo, Japan, July 2008, pp. 435-442.

[7] S. Niemann, J. Kotlarski, T. Ortmaier, and C. Muller-Schloer, "Reducing the optimization problem for the efficient motion planning of kinematically redundant parallel robots," in Proc. IEEE/ASME International Conference on Advanced Intelligent Mechatronics (AIM), Wollongong, Australia, 2013.

[8] S. Kucuk, "Energy minimization for 3-RRR fully planar parallel manipulator using particle swarm optimization," Mechanism and Machine Theory, vol. 62, no. 4, pp.129-149, 2013.

[9] A. Noshadi, M. Mailah, and Z. Ali, "Intelligent active force control of a 3-RRR parallel manipulator incorporating fuzzy resolved acceleration control," Journal of Applied Mathematical Modelling, vol. 36, pp. 2370-2383, 2012.

[10] Z. Gaoa, D. Zhanga, and Y. Geb, "Design optimization of a spatial six degree-of-freedom parallel manipulator based on artificial intelligence approaches," Robotics and Computer-Integrated Manufacturing, vol. 26, pp. 180-189, 2010.

[11] R. Boudreau and S. Nokleby, "Force optimization of kinematically-redundant planar parallel manipulators following a desired trajectory," Mechanism and Machine Theory, vol. 56, pp. 138-155, 2012.

[12] J. Wang and C. M. Gosselin, "Kinematic Analysis and Design of Kinematically Redundant Parallel Mechanisms," Journal of Mechanical Design, Trans. ASME, vol. 126, no. 1, pp. 109-118, 2004.

[13] F. Firmani and R. P. Podhorodeski, "Singularity analysis of planar parallel manipulators based on forward kinematic solutions," Mechanism and Machine Theory, vol. 44, pp. 1386-1399, 2009.

[14] T. Huang, M. Li, Z. X. Li, and D. G. Chetwynd, "Conceptual design and dimensional synthesis of a novel 2-DOF translational parallel robot for pick-and-place operations," Journal of Mechanical Design, Trans. ASME, vol. 126, no. 3, pp. 449-455, 2004

[15] K. Deb, Optimization for Design, $2^{\text {nd }}$ edition, Prentice Hall of India, New Delhi, 2012.

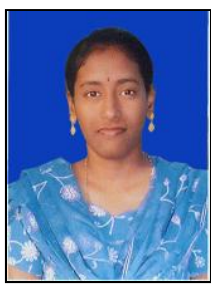

K. V. Varalakshmi was born in India. She received the $\mathrm{BE}$ degree in mechanical production and industrial engineering from Andhra University in 2006. And ME degree in CAD/CAM from Andhra University in 2009. Her current research interests include design and control of parallel manipulators.

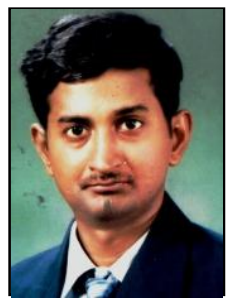

J. Srinivas is an associate professor in the Department of Mechanical Engineering, NIT Rourkela. His topics of interest include robotics and intelligent controls, dynamics and modeling. $\mathrm{He}$ guided various graduate and doctoral projects. He is a member of Institute of Engineers and has his credit around 80 papers published in various national and international conferences/journals. $\mathrm{He}$ is a main author of a book on robotics, control and programming published by Narosa Publishing house. 